\title{
Global and National Trends Toward Hepatitis in COVID-19 Era: Emerging Pandemic and Neglect of Other Diseases
}

\author{
Mohammad Reza Hedayati-Moghaddam (iD) ${ }^{1}$ and Reza Jafarzadeh Esfehani (iD) 1,* \\ ${ }^{1}$ Blood Borne Infections Research Center, Academic Center for Education, Culture and Research (ACECR), Razavi Khorasan Branch, Mashhad, Iran \\ "Corresponding author: Research Center, Academic Center for Education, Culture and Research (ACECR), Razavi Khorasan Branch, Mashhad, Iran. Email: jafarzadehr@jdm.ac.ir
} Received 2021 December 14; Revised 2021 December 27; Accepted 2021 December 27.

Keywords: Viral Hepatitis, COVID-19, Trend

The coronavirus disease 2019 (COVID-19) pandemic had significant mortality and morbidity for almost every healthcare system worldwide. During the first months of the pandemic, healthcare systems became overwhelmed, and numerous infected individuals suffered from a lack of medical resources. After introducing the first COVID19 vaccines, the disease became more controlled, and widespread global vaccination reduced the restrictions in numerous countries. Although many individuals have returned to their everyday lives, some adverse effects of the pandemic on public health are gradually becoming more prominent (1). Therefore, it seems that while we have stepped forward in overcoming the pandemic, we have stepped back in the management of other diseases.

Some other viral infectious diseases, including hepatitis $\mathrm{B}$ and $\mathrm{C}$, are among these diseases. The elimination of viral hepatitis as a public threat by 2030 has been determined as a global goal by World Health Organization (WHO) (2). Chronic hepatitis is a widely distributed disease worldwide, and its silent nature has made it remain undiagnosed. The prevention and control of widespread hepatitis require a multidisciplinary approach involving healthcare providers and patients (2).

However, during the COVID-19 pandemic, viral hepatitis seems to have become neglected in numerous countries, including Iran. This issue can be highlighted by using the "Google trends" evaluating the searches for the word "hepatitis" over the Internet (3). As shown in Figure 1 , the worldwide searches for "hepatitis" declined dramatically over March 2020, when the WHO declared COVID-19 as a pandemic (4). After about 20 months since the initiation of the pandemic, the mean count of search terms remained considerably low. However, by considering the mean searches for the words "SGOT", "SGPT", "bilirubin", and the "liver function test", there was a 2-month decline followed by an increase in the mean search over the next 18 months, probably due to impact of COVID-19 and its treatment on liver function tests (5).

Further investigations revealed that we are facing a similar trend in Iran over the past years (Figure 2). Such findings regarding the reduced global trend toward searching for hepatitis despite overall consistent search trends for liver enzymes could be alarming for healthcare providers. Such an issue about viral hepatitis during the COVID-19 has been previously addressed by Rehman et al., describing the impact of the pandemic on hepatitis B and hepatitis C prevention strategies (6). The prevention strategies, including vaccination, screening, diagnostic tests, and conducting awareness programs, have been negatively affected during the pandemic. Numerous patients refused to have routine vaccination or diagnostic tests due to fear of COVID-19. Moreover, numerous lowand middle-income countries did not provide a considerable number of awareness programs for the general population and patients with viral hepatitis during the pandemic $(7,8)$. On the other hand, many of the awareness and screening programs had been conducted in prevention and diagnostic facilities, faced restrictions during the pandemic, and numerous individuals who sought their diagnostic and screening programs could not receive healthcare services (7).

In conclusion, the COVID-19 pandemic had various adverse effects on healthcare systems, and some globally distributed diseases, including viral hepatitis, have been marginalized. Since the decreased search trends for hepatitis have been continued for more than 20 months since the pandemic and the trends seem to continue in the same vein in the future, it is needed to reconsider the national prevention and educational plans. Such alarming signs could be warning for increased cases of viral hepati- 


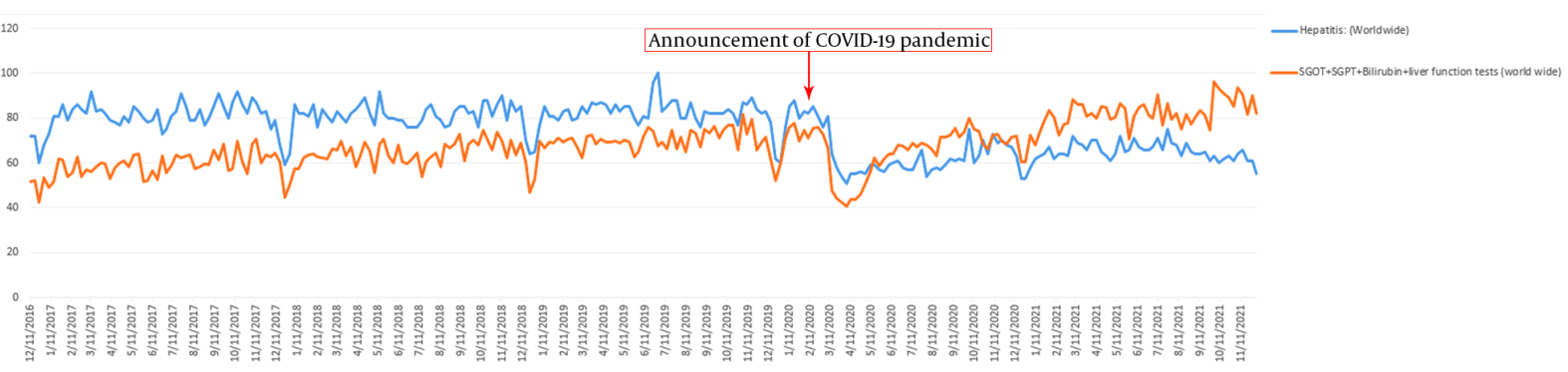

Figure 1. Mean number of global searches for hepatitis and liver enzyme tests during 5 years; Numbers on the vertical axis of the graph indicating search interest relative to the highest point on the chart over the specific time

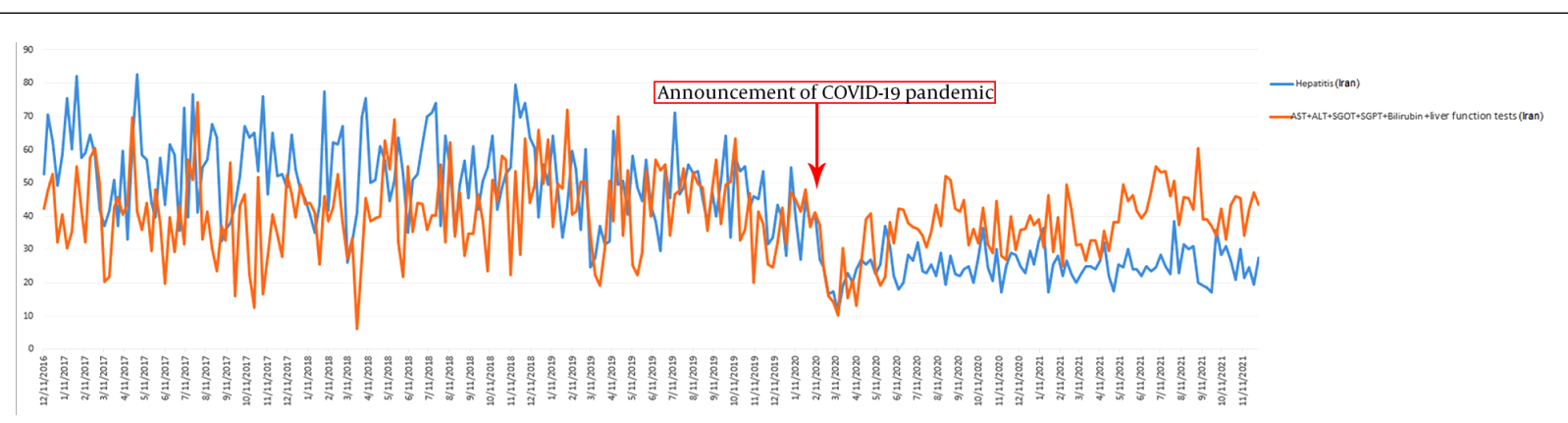

Figure 2. Mean number of searches for Persian equivalent words related to hepatitis and liver tests during 5 years in Iran; Numbers on the vertical axis of the graph indicating search interest relative to the highest point on the chart over the specific time

tis shortly and seek further studies to validate the trends and increase the national awareness simultaneously.

\section{Footnotes}

Authors' Contribution: R. JE wrote the manuscript with the valuable assistance of MR. HM.

Conflict of Interests: All the authors declare that there is no conflict of interest.

\section{References}

1. Gebru AA, Birhanu T, Wendimu E, Ayalew AF, Mulat S, Abasimel HZ, et al. Global burden of COVID-19: Situational analyis and review. Hum Antibodies. 2021;29(2):139-48. doi: 10.3233/HAB-200420. [PubMed: 32804122].

2. World Health Organization. Global hepatitis report. World Health Organization; 2017.
3. Google Inc. Google trends. Google Inc; 2021, [updated 11/12/2021]. Available from: https://trends.google.com/trends/?geo=IR.

4. Cucinotta D, Vanelli M. WHO declares COVID-19 a pandemic. Acta Bio Medica: Atenei Parmensis. 2020;91(1):157-60.

5. Bzeizi K, Abdulla M, Mohammed N, Alqamish J, Jamshidi N, Broering D. Effect of COVID-19 on liver abnormalities: a systematic review and meta-analysis. Sci Rep. 2021;11(1):10599. doi: 10.1038/s41598-021-895139. [PubMed: 34012016]. [PubMed Central: PMC8134580].

6. Rehman ST, Rehman H, Abid S. Impact of coronavirus disease 2019 on prevention and elimination strategies for hepatitis B and hepatitis $C$. World J Hepatol. 2021;13(7):781-9. doi: 10.4254/wjh.v13.i7.781. [PubMed: 34367499]. [PubMed Central: PMC8326164].

7. Toyoda H, Huang DQ, Le MH, Nguyen MH. Liver Care and Surveillance: The Global Impact of the COVID-19 Pandemic. Hepatol Commun. 2020;4(12):1751-7. doi: 10.1002/hep4.1579. [PubMed: 32838107]. [PubMed Central: PMC7405084].

8. Blach S, Kondili LA, Aghemo A, Cai Z, Dugan E, Estes C, et al. Impact of COVID-19 on global HCV elimination efforts. J Hepatol. 2021;74(1):31-6 doi:10.1016/j.jhep.2020.07.042. [PubMed:32777322]. [PubMed Central: PMC7411379]. 